\title{
Epigenetic changes in peripheral leucocytes as biomarkers in intrauterine growth retardation rat
}

\author{
XUE-FENG XU ${ }^{1}$, SHAN-SHAN XU ${ }^{2}$, LIN-CHENG FU ${ }^{2}$, QIONG-YAO HU ${ }^{2}$, YING LV $^{2}$ and LI-ZHONG DU ${ }^{2}$ \\ Departments of ${ }^{1}$ Respiratory Medicine and ${ }^{2}$ Neonatology, The Children's Hospital, \\ Zhejiang University School of Medicine, Hangzhou, Zhejiang 310052, P.R. China
}

Received June 13, 2016; Accepted September 22, 2016

DOI: $10.3892 /$ br.2016.775

\begin{abstract}
Epigenetics plays an important role in the fetal origins of adult disease. Intrauterine growth retardation (IUGR) can cause increased histone acetylation of the endothelin-1 (ET-1) gene from pulmonary vascular endothelial cells or the whole lung tissue and persist into later life, likely resulting in increased risk of pulmonary hypertension or asthma later in life. However, little is known regarding the correlation of epigenetic changes between specific tissue and peripheral leucocytes. In the present study, an IUGR rat model was established by maternal nutrient restriction. Peripheral blood leucocytes were isolated to detect the ET-1 expression level. Chromatin immunoprecipitation was used to analyze histone modification of the ET-1 gene promoter. The ET-1 protein expression of leucocytes from the 1-week IUGR group was similar to that from the 1 -week control group. ET-1 protein expression of leucocytes from 10-week IUGR rats was obviously higher than that of the other groups $(\mathrm{P}<0.05)$. The levels of acetylated histone $\mathrm{H} 3$ in the ET-1 promoter of leucocytes from the 1 -week IUGR rats were significantly higher than those from the age-matched control group $(\mathrm{P}=0.004)$. Furthermore, the trends continued $\leq 10$ weeks after birth. In conclusion, epigenetic modifications of leucocytes can in part reflect the epigenetic changes of lung tissue in IUGR rats. Epigenetics of peripheral leucocytes may be used as a biomarker for predicting the risk of the development of disease, and may be used as a surrogate to investigate the subsequent development of pulmonary vascular disease or asthma.
\end{abstract}

Correspondence to: Professor Li-Zhong Du, Department of Neonatology, The Children's Hospital, Zhejiang University School of Medicine, 3333 Binsheng Road, Hangzhou, Zhejiang 310052, P.R. China

E-mail: dulizhong@zju.edu..cn

Key words: epigenetics, leucocytes, endothelin-1, intrauterine growth retardation

\section{Introduction}

An adverse intrauterine environment may have an impact on the development of the fetus resulting in fetal intrauterine growth retardation (IUGR). IUGR accounts for $\geq 10 \%$ of all live births. Their perinatal mortality is 4 - to 10 -fold higher than that of normally grown babies $(1,2)$. Previous studies revealed that IUGR or low birth weight has been linked to the later development of diseases in adulthood, including type 2 diabetes, hypertension, and asthma (3-8). This is also known as the 'developmental origins of adult disease' theory or the Barker' hypothesis (9). Evidence suggests that epigenetics plays an important role in the fetal origin of adult disease (10). Epigenetics refers to all the heritable changes in phenotype or in gene expression states that are not involved in the DNA sequence itself. Histone modification and DNA methylation patterns are the predominant epigenetic phenomena, providing a mechanism that allows the stable propagation of gene activity states from one generation of cells to the next (11).

Previous findings showed that maternal nutrient restriction increased the histone acetylation and hypoxia inducible factor-1 $\alpha$ binding levels in the endothelin-1 (ET-1) gene promoter of pulmonary vascular endothelial cells (PVEC) in IUGR newborn rats, and continued $\leq 6$ weeks after birth (12). However, this intervention did not result in a change in ET-1 gene methylation. These epigenetic changes resulted in an IUGR rat being highly sensitive to hypoxia later in life, causing more significant pulmonary arterial hypertension or pulmonary vascular remodeling (12). Intrauterine nutrient restriction may, not only cause increased histone acetylation of the ET-1 gene at the endothelial cellular level, but cause corresponding changes of the ET-1 gene at a whole lung tissue level and persist into later life. The effect persisted up to 10 weeks after birth. These epigenetic changes may induce an IUGR individual to be highly sensitive to OVA (allergen) challenge later in life, resulting in more significant changes related to asthma (13). The above mentioned findings suggested that epigenetics is closely associated with the development of pulmonary arterial hypertension and asthma following IUGR.

Increased histone acetylation levels of ET-1 gene in lung tissue following IUGR can cause IUGR individual susceptibility to hypoxia stimulation or allergen challenge. This epigenetic susceptibility following IUGR resulted in the development of adulthood disease later in life, involving 
pulmonary hypertension and asthma. It is not fully understood whether the epigenetic susceptibility in lung tissue can be found at an early stage. In view of ethics, it is almost impossible to obtain a biopsy of the lung tissue to detect the corresponding epigenetic changes. Of note, the adverse intrauterine environment or prenatal stress may, not only cause disease-specific or tissue-specific epigenetic modifications but also induce epigenetic changes of peripheral leucocytes $(14,15)$. It further indicated whether maternal nutrient restriction may not only cause epigenetic changes of lung tissue, but also epigenetic changes of peripheral leucocytes.

Therefore, we speculated that IUGR did not only induce epigenetic modifications of ET-1 gene in lung tissue, but also caused epigenetic modifications in peripheral leucocytes. In the present study, histone acetylation levels of the ET-1 gene in leucocytes from 1-and 10-week IUGR rats were investigated.

\section{Materials and methods}

IUGR rat model. All the procedures and protocols were approved by the Animal Care and Use Committee of Zhejiang University (approval no. zju201305-1-02-043). The IUGR rat model was established based on our previous study (12). Briefly, virgin female Sprague-Dawley rats weighing 250-300 g were mated overnight. After confirmation of mating, the pregnant rats were randomly divided into two nutritional groups: i) Standard diet ad libitum throughout gestation (control group), and ii) undernutrition group (IUGR). Pregnant rats in the undernutrition group were fed the same diet at $50 \%$ of the free intake until birth. The two groups of rats were kept in the same room with a constant temperature maintained at $22 \pm 3^{\circ} \mathrm{C}$, and had free access to water. Pups whose birth weight was below the $10 \%$ of normal birth weight, were defined as IUGR rats. Newborn IUGR rats continued to be reared by diet-restricted mothers that received normal food intake through lactation, while the control pups were reared by control mothers. Rats of both groups were raised up to 10 weeks of age.

Isolation of peripheral blood leucocytes. Whole blood samples were drawn from the rats via abdominal aorta and collected into lithium heparin tubes. Peripheral blood leucocytes were isolated according to the manufacturer instructions (1083-1; Sigma, St. Louis, MO, USA). Briefly, 3 ml Histopaque 1083 was added to a $15 \mathrm{ml}$ centrifuge tube, and $3.0 \mathrm{ml}$ whole blood was carefully layered onto the Histopaque 1083 surface, which was then centrifuged at $400 \mathrm{x} \mathrm{g}$ for $30 \mathrm{~min}$ at room temperature. After centrifugation, 2-3 $\mathrm{mm}$ of the opaque layer containing the leucocytes was aspirated, and the opaque interface containing the leucocytes band was transferred into a clean $15 \mathrm{ml}$ conical centrifuge tube. Isotonic PBS $(10 \mathrm{ml})$ was added to the tube, and the tube was mixed by gentle inversion several times. Tubes were centrifuged at $250 \mathrm{x}$ g for $10 \mathrm{~min}$, and the supernatant was discarded. After washing with PBS, the cell pellet was kept at $-80^{\circ} \mathrm{C}$ until use.

ET-1 expression in leucocytes. Total protein extracts and protein concentrations were prepared according to our previous studies $(16,17)$. A total of $30 \mu \mathrm{g}$ of protein extracts were resolved on $8-10 \%$ SDS-polyacrylamide gel electrophoresis for ET-1 protein. Proteins were transferred onto a polyvinylidene fluoride membrane using a Bio-Rad gel blotting apparatus (Bio-Rad Laboratories, Hercules, CA, USA). The membranes were incubated with a primary antibody against ET-1 overnight at $4^{\circ} \mathrm{C}$ and with a peroxidase-conjugated secondary antibody for $60 \mathrm{~min}$ at room temperature. Peroxidase was visualized by enhanced chemiluminescence (ECL) and exposure to ECL films for appropriate times. The bands of western blot analyses were quantified by densitometry and normalized with $\beta$-actin using Image-Pro Plus software. The protein level was expressed as a percentage of control 1 week \pm standard error of the mean (SEM).

Quantitative polymerase chain reaction (qPCR) for ET-1 was also performed. Total RNA was isolated from leucocytes according to the RNeasy protocol (Axygen, Tewksbury, MA, USA). Primers used for ET- 1 and $\beta$-actin were: Forward: 5'-aag cagacaaagaactccgag-3' and reverse: 5'-cgctttcaactttgcaa ctcg-3'; forward: 5'-gccaaccgtgaaaagatg-3' and reverse: 5'-tgccagtggta cgaccag-3', respectively (12).

Chromatin immunoprecipitation (ChIP) assay. ChIP assay was performed as described in a previous study (17). After the isolated leucocytes were fixed and cross-linked, the cell pellets were suspended in cell lysis buffer and centrifuged, and the supernatants were discarded. The pellets were resuspended in SDS lysis buffer, sonicated, centrifuged and aliquots of soluble chromatin were collected. Aliquots of the supernatants were retained for representing the input chromatin. Aliquots were incubated overnight at $4^{\circ} \mathrm{C}$ with one of the following antibodies: $5 \mu \mathrm{l}$ anti-acetylaled histone H3 (06-599; Merck Millipore, Billerica, MA, USA), $5 \mu$ l anti-acetylated histone H3 (H3K9/18) (07-593; Merck Millipore), and $5 \mu$ l anti-acetylaled histone H4 (06-866; Merck Millipore). The immune complexes were precipitated with protein A beads and then eluted. The input, unbound and bound fractions were incubated with $5 \mathrm{M} \mathrm{NaCl}$ to reverse crosslink. The DNA fragments were purified using the Qiaquick PCR Purification kit (Qiagen, Hilden, Germany). The DNA fragments containing ET-1 site-specific sequences were quantified by qPCR. Relative quantification of PCR products was based on value differences between the bound and input using the $\triangle \Delta \mathrm{Cq}$ method. The PCR primers for the ET1 gene promoter were as follows: ET-1 promoter A1, forward: 5'-ttgcctgtgggtgactaatc-3' and reverse: 5'-ccttcaccggag cgaaag-3' (-197 to +25); ET-1 promoter A2, forward: 5'-cctctt gattcttgaactctggg-3' and reverse: 5'-attagtcacccacaggcaac-3' (-397 to -179$)$.

Statistical analysis. Values are expressed as mean \pm SEM. The continuous variables in the different groups were compared by one-way ANOVA or t-test. $\mathrm{P}<0.05$ was considered to indicate a statistically significant difference.

\section{Results}

ET-1 protein expression of leucocytes. Unlike the higher expression of ET-1 in endothelial cells or lung tissue, ET-1 protein expression in peripheral leukocytes was relatively low (Fig. 1). The ET-1 protein level of leucocytes from the 1-week IUGR group was slightly high compared with that 
A

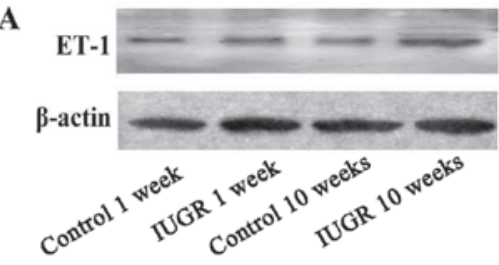

B
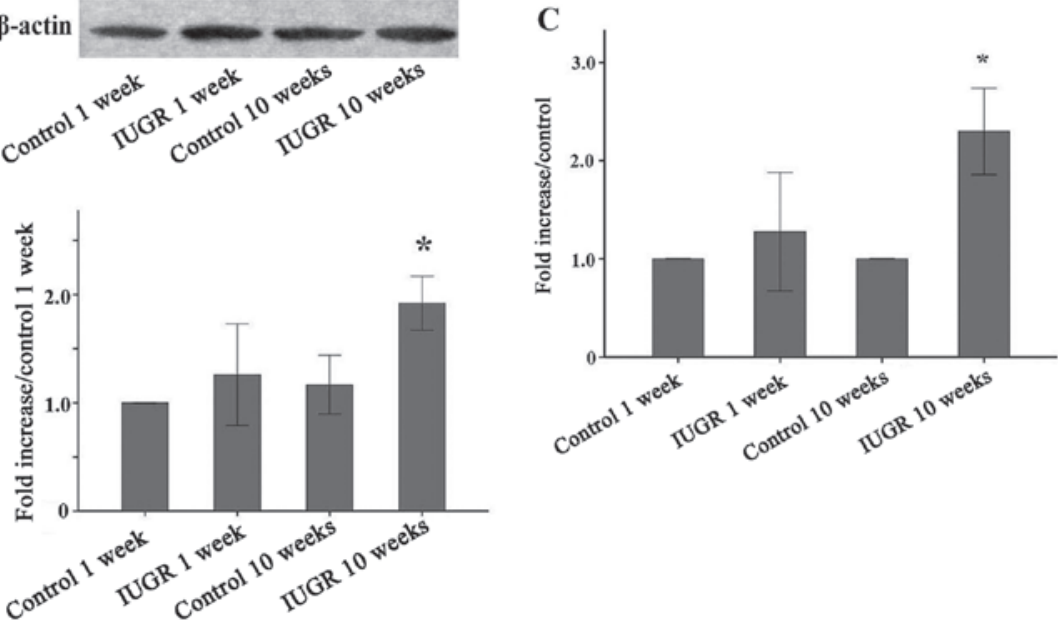

Figure 1. Quantification and representative western blots. Peripheral leucocyte protein extractions from control 1 week, IUGR 1 week, control 10 weeks, and IUGR 10 weeks group rats were used for the western blot analysis of ET-1 protein expression. (A) Representative western blot graphs are shown, $\beta$-actin protein expression serves as an internal control and is used to normalize the protein band intensity. (B) The bar graph shows the ET-1 protein relative levels (percent of control 1 week \pm SEM). (C) ET-1 mRNA levels. Increased expression of the ET-1 protein and mRNA in peripheral leucocytes from IUGR 10 weeks. IUGR, intrauterine growth retardation; ET-1, endothelin-1; SEM, standard error of the mean.

A

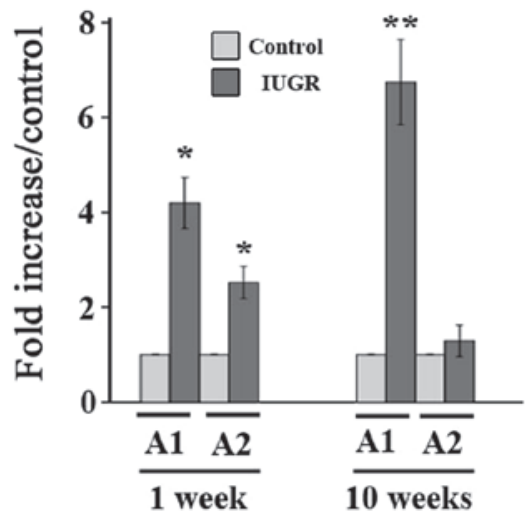

B

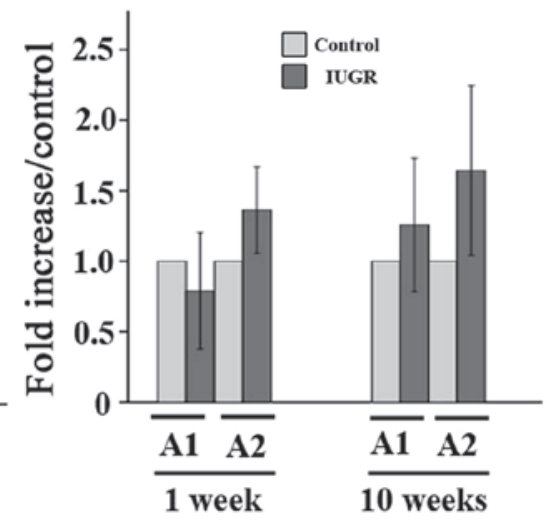

Figure 2. Comparison of (A) histone $\mathrm{H} 3$ and (B) H3K9/18 acetylation levels at the rat ET-1 promoter from peripheral leucocytes between IUGR and the control using ChIP and relative quantitative polymerase chain reaction. A1 and A2 are two areas of the ET-1 gene promoter (-197 to +25 and -397 to -179$)$, respectively. Data are expressed as IUGR percent of the control $\pm \mathrm{SEM}$. " $\mathrm{P}<0.05$ as compared with the control groups, ${ }^{* *} \mathrm{P}<0.01$ as compared with the control groups, respectively. IUGR, intrauterine growth retardation; ET-1, endothelin-1; ChIP, chromatin immunoprecipitation.

from the 1-week control group. There was no statistically significant difference between the groups $(\mathrm{P}=0.113)$. However, ET-1 expression of leucocytes from the 10-week IUGR rats was obviously higher than that of the other groups $(\mathrm{P}<0.05)$. A similar trend was also observed in the ET-1 mRNA levels in leucocytes.

ET-1 gene promoter histone code of leucocytes. Two sites along the ET-1 promoter were analyzed for acetylated H3, H3 (Lys 9/18), and H4. The levels of acetylated histone $\mathrm{H} 3$ in the ET-1 promoter A1 region of leucocytes from the 1-week IUGR rats were significantly higher than those from the age-matched control group ( $\mathrm{P}=0.004$, Fig. 2). A similar change was also seen in the ET-1 promoter A2 region from 1-week IUGR rats. Furthermore, these trends continued $\leq 10$ weeks after birth. The levels of acetylated histone H3 (Lys 9/18) in the ET-1 promoter
A1 and A2 regions from 1-week IUGR rats were similar to the age-matched control groups $(\mathrm{P}>0.05)$. Although the levels of acetylated histone $\mathrm{H} 3$ (Lys 9/18) in the ET-1 promoter regions from the 10-week IUGR group were higher than those of the age-matched control group, there was no significant difference $(\mathrm{P}>0.05)$. Similar to the changes of acetylated histone $\mathrm{H} 4$ in PVEC or lung tissue $(12,13)$, there was no significant difference in the $\mathrm{H} 4$ acetylation levels of the ET-1 promoter regions from leucocytes between the IUGR and control groups.

\section{Discussion}

The present findings have shown that maternal nutrient restriction, not only increased the histone acetylation levels in the ET-1 gene promoter of PVEC or lung tissue, but also increased histone acetylation levels in the ET-1 gene of leucocytes from 
1-week IUGR rats. The effect was continued up to 10 weeks after birth. The epigenetic modifications of lung tissue ET-I gene were in part reflected via the epigenetic changes of peripheral blood leucocytes.

Increasing evidence suggests that aberrant epigenetic modifications of peripheral leucocytes are involved in autoimmune diseases or inflammatory disorders, cancer, and even Alzheimer's disease (18-20). DNA methylation from whole blood samples is a powerful and highly informative biomarker, not only for current smoking, but also for a lifetime history of smoking. Smoking-related methylation signatures may also be very useful predictors of smoking-associated risks (21). Global $\mathrm{H} 3$ acetylation and $\mathrm{H} 3 \mathrm{~K} 4$ methylation levels were significantly increased in peripheral blood mononuclear cells (PBMCs) from Henoch-Schönlein purpura patients with kidney damage in comparison with those patients without kidney involvement. These changes were positively correlated with the clinical HSP score, providing further evidence for the role of histone modifications in autoimmune diseases (22). On the other hand, global histone $\mathrm{H} 4$ and histone $\mathrm{H} 3$ acetylation levels were significantly decreased in PBMCs of patients with pemphigus vulgaris. Histone acetylation modifications are also aberrant in PBMCs of patients with Graves' disease (20). Different from the above studies mainly focusing on global histone modifications or DNA methylation in PMBCs, the present study focused on the epigenetic changes of single ET-I gene, possibly better predicting the risk of disease.

As the newborn rats were too small to obtain sufficient peripheral leucocytes, we utilized 1-week rats to represent the newborn rats. We found that, except histone H3K9/18, histone $\mathrm{H} 3$ and $\mathrm{H} 4$ acetylation levels of the ET-1 promoter in peripheral leucocytes from 1-week rats were similar to those in PVEC or lung tissue $(12,13)$. This trend persisted until 10 weeks after birth, indicating that the epigenetic modifications of peripheral leucocytes partly represent epigenetic changes of lung tissue. It is revealed that IUGR, not only induces tissue-specific epigenetic changes, but also epigenetic modifications of peripheral leucocytes. In a previous persistent pulmonary hypertension of the newborn rat, short-term hypoxia and indomethacin treatments in uterine were not sufficient to cause significant $\mathrm{CpG}$ methylation in the endothelial nitric oxide synthase (eNOS) gene promoter (17). Similarly, maternal nutrient restriction was insufficient to induce significant methylation changes in the ET-1 promoter, regardless of whether in PVEC or in lung tissue $(12,13)$. Although the methylation status of $\mathrm{CpG}$ islands of other genes were not analyzed, intrauterine different environments did not cause methylation changes of the eNOS or ET-1 gene. Therefore it is likely that intrauterine different environments may not induce methylation changes of the ET-1 gene in other tissue. In view of these findings, methylation modifications of CpG islands in ET-1 gene from peripheral leucocytes were not investigated in the present study.

Epigenetic changes of ET-1 gene in lung tissue and peripheral leucocytes may induce IUGR individuals later in life to be highly sensitive to hypoxia or allergen stimulations, resulting in more significant pulmonary hypertension and asthma-like manifestations. Epigenetic regulation of the ET-1 gene in lung tissue may be directly involved in the development of pulmonary arterial hypertension or asthma later in life. However, epigenetics of the ET-1 gene from peripheral leucocytes is not directly associated with the development of adulthood diseases. This finding further suggests that the epigenetic changes of peripheral leucocytes may be useful as a biomarker to predict the risk of development of pulmonary hypertension or asthma following IUGR. Nevertheless, caution is necessary when attempting to apply information from a rat model to human pathophysiology. The newborn and juvenile rats are physiologically immature relative to the human, and the insult imposed on the fetal rat in this model of nutrient restriction is obvious. In comparison, the effect of nutrient restriction on humans ranges across a continuum.

In conclusion, the present findings have shown aberrant histone acetylation modification of peripheral leucocytes in IUGR rats. The epigenetic modifications of leucocytes can in part reflect epigenetic changes of lung tissue, providing novel insights into understanding of the involvement of epigenetics in the fetal origin of adult disease. Furthermore, the epigenetics of peripheral leucocytes may be used as a biomarker for predicting the risk of the development of disease.

\section{Acknowledgements}

We would like to thank Zhejiang Key Laboratory for Diagnosis and Therapy of Neonatal Diseases, Key Laboratory of Reproductive Genetics Ministry of Education, and Bioelectromagnetics Laboratory of Zhejiang University School of Medicine for their assistance. The study was supported by grants from the National Natural Science Foundation of China (nos. 81270723, 81270722 and 81471480), Zheng Shu Medical Elite Scholarship Fund and Zhejiang Provincial Health High-Level Personnel Training Project of China (Yi Tan Xin Xiu).

\section{References}

1. Gardosi JO, Mongelli JM and Mul T: Intrauterine growth retardation. Baillieres Clin Obstet Gynaecol 9: 445-463, 1995.

2. Chiswick ML: Intrauterine growth retardation. Br Med J (Clin Res Ed) 291: 845-848, 1985.

3. Vickers MH, Breier BH, Cutfield WS, Hofman PL and Gluckman PD: Fetal origins of hyperphagia, obesity, and hypertension and postnatal amplification by hypercaloric nutrition. Am J Physiol Endocrinol Metab 279: E83-E87, 2000.

4. Bogdarina I, Welham S, King PJ, Burns SP and Clark AJL: Epigenetic modification of the renin-angiotensin system in the fetal programming of hypertension. Circ Res 100: 520-526, 2007.

5. Joss-Moore LA and Lane RH: The developmental origins of adult disease. Curr Opin Pediatr 21: 230-234, 2009.

6. Park JH, Stoffers DA, Nicholls RD and Simmons RA: Development of type 2 diabetes following intrauterine growth retardation in rats is associated with progressive epigenetic silencing of Pdx1. J Clin Invest 118: 2316-2324, 2008.

7. Bassan H, Trejo LL, Kariv N, Bassan M, Berger E, Fattal A, Gozes I and Harel S: Experimental intrauterine growth retardation alters renal development. Pediatr Nephrol 15: 192-195, 2000.

8. Xu XF, Li YJ, Sheng YJ, Liu JL, Tang LF and Chen ZM: Effect of low birth weight on childhood asthma: A meta-analysis. BMC Pediatr 14: 275, 2014.

9. Barker DJP: Fetal and infant origins of adult disease. Monatsschr Kinderheilkd 149: S2-S6, 2001.

10. Cutfield WS, Hofman PL, Mitchell M and Morison IM: Could epigenetics play a role in the developmental origins of health and disease? Pediatr Res 61: 68R-75R, 2007.

11. Xu XF and Du LZ: Epigenetics in neonatal diseases. Chin Med J (Engl) 123: 2948-2954, 2010. 
12. Xu XF, Lv Y, Gu WZ, Tang LL, Wei JK, Zhang LY and Du LZ Epigenetics of hypoxic pulmonary arterial hypertension following intrauterine growth retardation rat: epigenetics in $\mathrm{PAH}$ following IUGR. Respir Res 14: 20, 2013.

13. Xu XF, Hu QY, Liang LF, Wu L, Gu WZ, Tang LL, Fu LC and Du LZ: Epigenetics of hyper-responsiveness to allergen challenge following intrauterine growth retardation rat. Respir Res 15: 137, 2014.

14. Schlinzig T, Johansson S, Gunnar A, Ekström TJ and Norman M: Epigenetic modulation at birth - altered DNA-methylation in white blood cells after Caesarean section. Acta Paediatr 98 1096-1099, 2009.

15. Oberlander TF, Weinberg J, Papsdorf M, Grunau R, Misri S and Devlin AM: Prenatal exposure to maternal depression, neonatal methylation of human glucocorticoid receptor gene (NR3C1) and infant cortisol stress responses. Epigenetics 3: 97-106, 2008.

16. Xu XF, Gu WZ, Wu XL, Li RY and Du LZ: Fetal pulmonary vascular remodeling in a rat model induced by hypoxia and indomethacin. J Matern Fetal Neonatal Med 24: 172-182, 2011.

17. Xu XF, Ma XL, Shen Z, Wu XL, Cheng F and Du LZ: Epigenetic regulation of the endothelial nitric oxide synthase gene in persistent pulmonary hypertension of the newborn rat. J Hypertens 28: 2227-2235, 2010.
18. Hou Y, Chen H, He Q, Jiang W, Luo T, Duan J, Mu N, He Y and Wang H: Changes in methylation patterns of multiple genes from peripheral blood leucocytes of Alzheimer's disease patients. Acta Neuropsychiatr 25: 66-76, 2013.

19. Bauden M, Pamart D, Ansari D, Herzog M, Eccleston M, Micallef J, Andersson B and Andersson R: Circulating nucleosomes as epigenetic biomarkers in pancreatic cancer. Clin Epigenetics 7: 106, 2015.

20. Yan N, Zhou JZ, Zhang JA, Cai T, Zhang W, Wang Y, Muhali FS, Guan L and Song RH: Histone hypoacetylation and increased histone deacetylases in peripheral blood mononuclear cells from patients with Graves' disease. Mol Cell Endocrinol 414: 143-147, 2015.

21. Gao X, Jia M, Zhang Y, Breitling LP and Brenner H: DNA methylation changes of whole blood cells in response to active smoking exposure in adults: A systematic review of DNA methylation studies. Clin Epigenetics 7: 113, 2015.

22. Luo S, Liang G, Zhang P, Zhao M and Lu Q: Aberrant histone modifications in peripheral blood mononuclear cells from patients with Henoch-Schönlein purpura. Clin Immunol 146: 165-175, 2013. 